Article

\title{
The Geomagnetic Field (GMF) Modulates Nutrient Status and Lipid Metabolism during Arabidopsis thaliana Plant Development
}

\author{
Monirul Islam (D), Gianpiero Vigani and Massimo E. Maffei *(D) \\ Plant Physiology Unit, Department of Life Sciences and Systems Biology, University of Turin, 10135 Turin, Italy; \\ monirul.islam@unito.it (M.I.); gianpiero.vigani@unito.it (G.V.) \\ * Correspondence: massimo.maffei@unito.it; Tel.: +39-011-670-5967
}

Received: 27 October 2020; Accepted: 4 December 2020; Published: 8 December 2020

check for updates

\begin{abstract}
The Geomagnetic field (GMF) is a typical component of our planet. Plant perception of the GMF implies that any magnetic field (MF) variation would induce possible metabolic changes. In this work was we assessed the role of the GMF on Arabidopsis thaliana Col0 mineral nutrition and lipid metabolism during plant development. We reduced the local GMF (about $40 \mu \mathrm{T}$ ) to Near Null Magnetic Field (NNMF, about $30 \mathrm{nT}$ ) to evaluate the effects of GMF on Arabidopsis in a time-course (from rosette to seed-set) experiment by studying the lipid content (fatty acids, FA; and surface alkanes, SA) and mineral nutrients. The expression of selected genes involved in lipid metabolism was assessed by Real-Time PCR (qPCR). A progressive increase of SA with carbon numbers between 21 and 28 was found in plants exposed to NNMF from bolting to flowering developmental stages, whereas the content of some FA significantly $(p<0.05)$ increased in rosette, bolting and seed-set developmental stages. Variations in SA composition were correlated to the differential expression of several Arabidopsis 3-ketoacyl-CoAsynthase (KCS) genes, including KCS1, KCS5, KCS6, KCS8, and KCS12, a lipid transfer protein (LTPG1) and a lipase (LIP1). Ionomic analysis showed a significant variation in some micronutrients ( $\mathrm{Fe}, \mathrm{Co}, \mathrm{Mn}$ and $\mathrm{Ni}$ ) and macronutrients $(\mathrm{Mg}, \mathrm{K}$ and $\mathrm{Ca}$ ) during plant development of plants exposed to NNMF. The results of this work show that $A$. thaliana responds to variations of the GMF which are perceived as is typical of abiotic stress responses.
\end{abstract}

Keywords: Arabidopsis thaliana; Helmholtz coils; gene expression; fatty acid content; surface alkane content; micronutrients; macronutrients; near null magnetic field

\section{Introduction}

As sessile organisms, plants have evolved both constitutive and inducible responses to the changing environment. Several environmental factors affect plant growth and developments. Among them, the Earth magnetic field or geomagnetic field (GMF, about $40 \mu \mathrm{T}$ ) is an environmental component of our planet and its reduction to Near Null Magnetic Field (NNMF, about $30 \mathrm{nT}$ ) has been shown to influence many plant biological processes [1,2]. One of the major effects of GMF reduction is the delay in flowering time and the alteration of clock gene amplitude clock [3-5]. Growing plants in NNMF condition also alters the plant mineral nutrition, through modulating channels, transporters and genes involved in mineral absorption and assimilation [6,7]. Three different mechanisms of magnetoperception have been described: (1) a mechanism involving radical pairs (i.e., magnetically sensitive chemical intermediates that are formed by photoexcitation of cryptochrome [8]), which has been demonstrated in plants [9]; (2) the presence of magnetic field (MF) sensory receptors present in cells containing ferromagnetic particles, as has been shown in magnetotactic bacteria [10]; and (3) the detection of minute electric fields by electroreceptors in the ampullae of Lorenzini in elasmobranch animals [11]. 
Plants show both light-dependent $[9,12,13]$ and light-independent $[3,14]$ magnetoreception, which may reflect a differential ability of plant organs to interact with light and the GMF. Cryptochromes which are blue-light photoreceptors are involve in magnetoreception and the effect of magnetic fields was reported in cryptochrome phosphorylation [9,15-17]. Phytochromes, the red-light photoreceptors, are known to interact with cryptochrome at many levels and enhances and maintains plant downstream responses to blue light [18]. Phytochrome phyAphyB deficient mutants showed a visibly enhanced response to an applied MF [9]. Therefore, plants can use different cues to perceive variations in the MF and triggers signal transduction events eventually leading to biochemical and developmental changes.

Lipids play crucial roles in plant development being both basic components of cellular membranes and constituents of suberin and cutin waxes that provide structural barriers to the environment $[19,20]$. They contribute to inducible stress resistance through the remodeling of membrane fluidity [21]. Other than their structural and functional features, lipids play also important roles as signaling and regulatory molecules in cell biology. In plants, several physiological processes linked to plant growth and plant responses to environmental stimuli are under control of a complex network of lipid-mediated signaling [22]. Variations of MF have been found to alter the composition and content of radish and onion polar and neutral lipids and the composition of their fatty acids [23-25] affecting lipid synthesis in chloroplast, mitochondrial, and other cell membranes [26,27]. The epicuticular layers, which are mainly composed by linear and branched alkanes derived from FA elongation and decarbonilation, are involved in the external cell barrier against the entry of pathogens and the limitation of transpiration [28].

In order to investigate the role of the GMF on both lipid metabolism and growth of Arabidopsis plants during development, we exposed plants to NNMF conditions. Here we show that the reduction of the GMF to NNMF affects plant nutrition by altering the content of both micro and micronutrients and the lipid content and composition of Arabidopsis by a differential modulation of several 3-ketoacyl-CoAsynthase (KCS) genes, a lipid transfer protein (LTPG1), a lipase (LIP1), and a fatty acid desaturase (ADS4).

\section{Results and Discussion}

\subsection{The GMF Modulates Wax Alkane Production during Plant Development}

In plant exposed to NNMF we found a progressive increase of cuticular wax alkanes from the early stages of plant development (rosette) to the late phase of plant growth before senescence (seed-set), when compared to GMF (Figure 1). In general, we identified several cuticular wax alkanes with a chain length ranging from 21 to 31 carbon atoms (see material and methods for alkane nomenclature). Our results are in line with the general composition of Arabidopsis wax alkanes, with molecules ranging from 21 to 29 carbon atoms [20,29]. In particular, in the rosette stage of development (See Supplementary Figure S1), exposure of plants to NNMF induced a significant $(p<0.05)$ reduction in the content of almost all identified alkanes (with the sole exception of C31) (Figure 1A). The major alkane at this stage of development was C25 in GMF exposed plants, whereas in NNMF exposed plants C26, C27 and C31 were more abundant (Figure 1A). In the bolting stage of development (Supplementary Figure S1), the major alkanes were C29 and C31 in both GMF and NNMF exposed plants, which showed no quantitative significant $(p>0.05)$ differences in the content of these alkanes (Figure 1B). However, significant ( $p<0.05$ ) increases of C22, C24, C25, C26, C27, C28, and C30 were observed in plants exposed to NNMF with respect to GMF control plants (Figure 1B). During the flowering stage of development (Supplementary Figure S1), a strong and significant increase for most of the alkanes was found in plant exposed to NNMF with respect to GMF control plants, with the sole exception for C30, which was more abundant in GMF plants (Figure 1C). Finally, in the seed-set stage of development (at 35 DAS, Supplementary Figure S1), both NNMF and GMF exposed plants showed a drastic reduction of alkanes from C21 to C30 (Figure 1D). The only remaining alkanes were C29 and C31, which were significantly $(p<0.05)$ higher in NNMF plant with respect to GMF control plants (Figure 1D). 

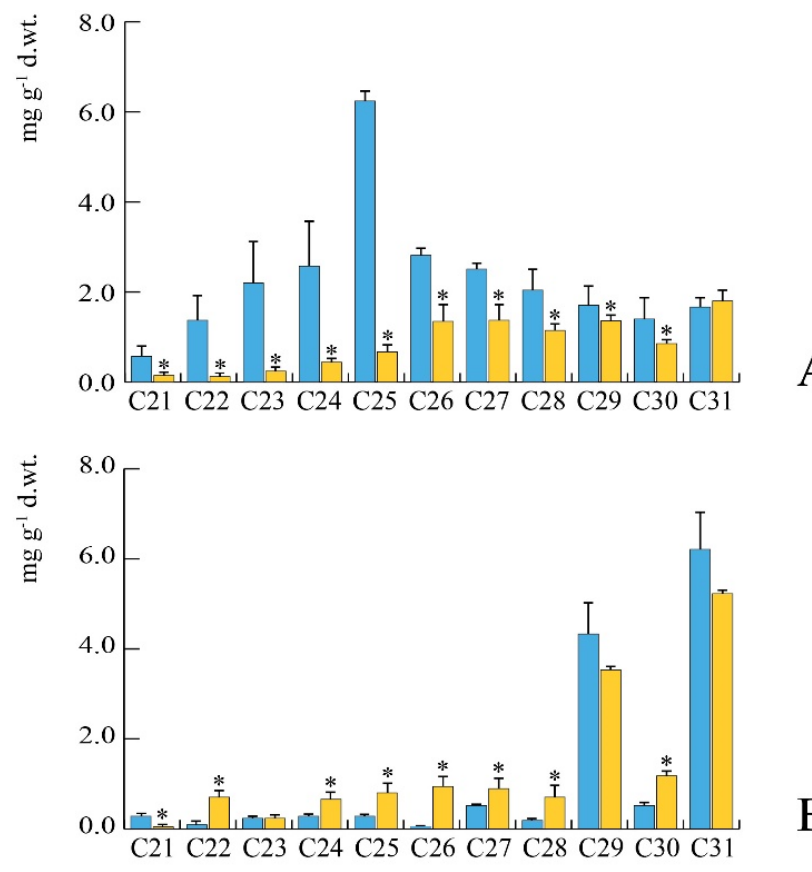

B

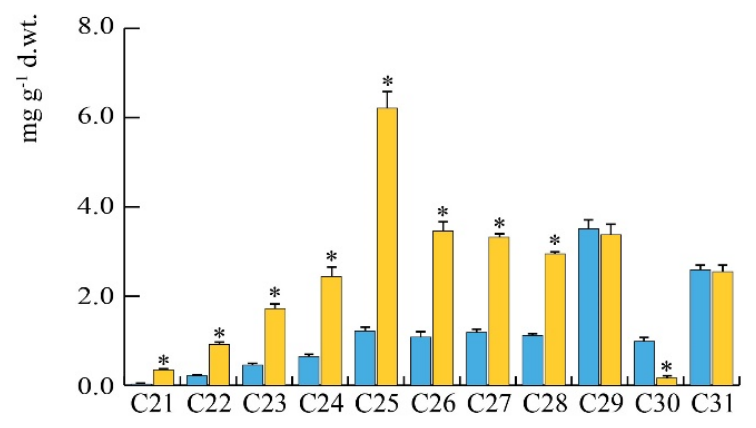

$\mathrm{C}$

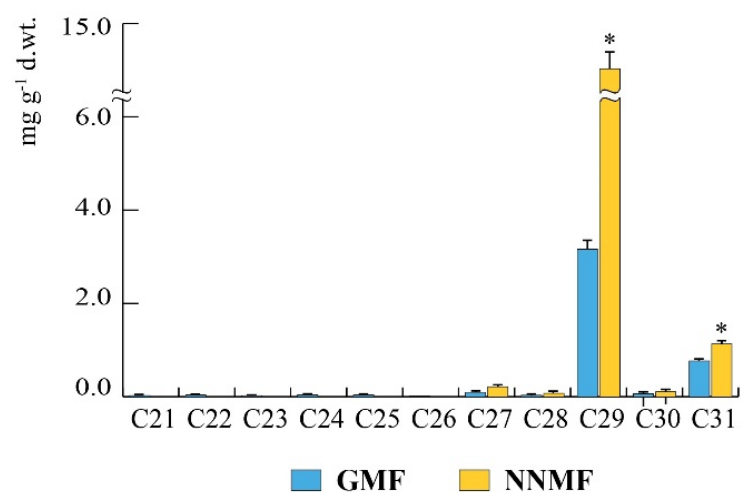

D

Figure 1. Surface alkane composition during Arabidopsis thaliana development upon exposure to either geomagnetic field (GMF, blue bars) or near-null magnetic field (NNMF, yellow bars). (A), rosette developmental stage, 15 days old plants. (B), bolting developmental stage, 21 days old plants. (C), flowering developmental stage, 30 days old plants. (D), seed-set developmental stage, 35 days old plants. Bars represent standard deviation. *, significant $(p<0.05)$ differences between NNMF and GMF plants.

\subsection{The GMF Modulates Fatty Acid Production during Plant Development}

Several fatty acids (FAs) were affected by the reduction of the GMF, including palmitic (C16:0), palmitoleic (C16:1), stearic (C18:0), oleic (C18:1), linoleic (C18:2) and linolenic (C18:3) acids (Figure 2). In the rosette stage of development, NNMF plants showed a significantly $(p<0.05)$ higher content 
of C18:2 with respect to GMF plants, whereas no significant difference was found for the other FAs (Figure 2A). Almost the same results were found in the bolting stage of development, but in this case NNMF plants showed also a significant $(p<0.05)$ increase of C18:1 and a decrease of C18:0, with respect to GMF plants (Figure 2B). In the flowering stage, NNMF plants showed a significant $(p<0.05)$ reduction of $\mathrm{C} 18: 2$ with respect to GMF plants (Figure $2 \mathrm{C}$ ), whereas in the seed-set stage of development the C16:1 and C18:1 of NNMF exposed plants showed a significant $(p<0.05)$ increase with respect to GMF plants (Figure 2D). The increase of 18:1 and 18:2 and the reduction of 18:0 observed in our study are in line with the effects observed in other plants exposed to reduced MF $[24,27]$.

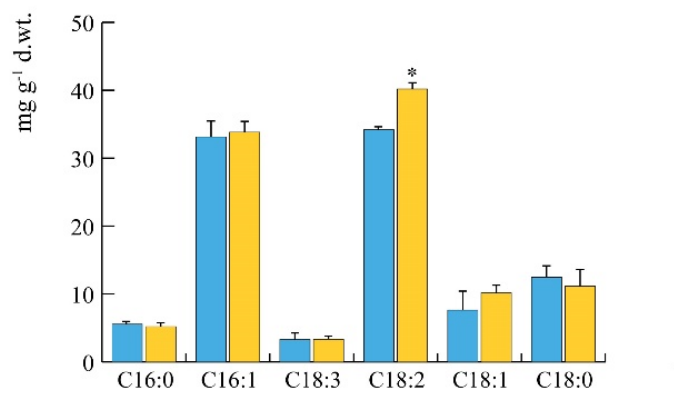

A

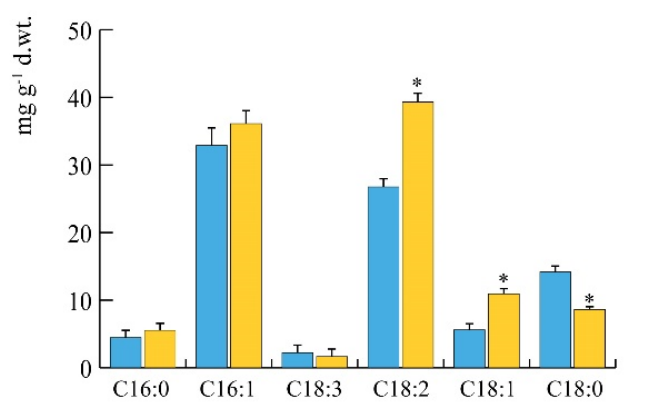

B

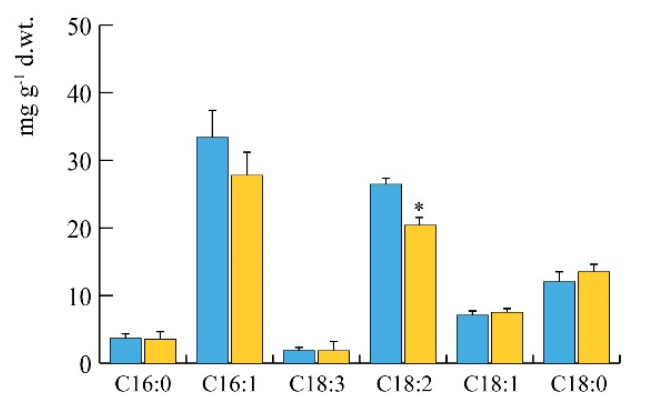

C

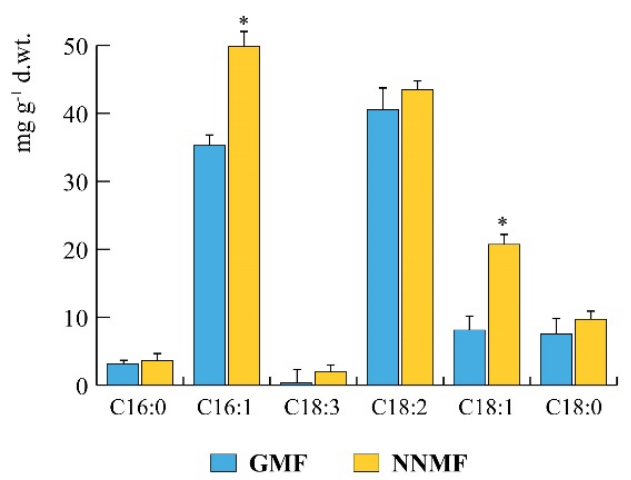

D

Figure 2. Developmental changes in fatty acid composition of Arabidopsis thaliana exposed to geomagnetic field (GMF, blue bars) or to near-null magnetic field (NNMF, yellow bars). (A), rosette developmental stage, 15 days old plants. (B), bolting developmental stage, 21 days old plants. (C), flowering developmental stage, 30 days old plants. (D), seed-set developmental stage, 35 days old plants. Bars represent standard deviation. *, significant $(p<0.05)$ differences between NNMF and GMF plants. 


\subsection{The GMF Modulates the Expression of Lipid-Realted Genes during Plant Development}

Among the several genes that are involved in lipid metabolism we selected some genes involved in fatty acids and alkane metabolism. Figure 3 shows the fold-change gene expression of Arabidopsis plants exposed to NNMF with respect to control plants exposed to GMF during plant development. In the rosette stage of development, upregulation of LTPG1, KCS1, and KCS12 was correlated to the NNMF-dependent increased content of C18:2 (see Figure 2A), whereas the downregulation of KCS5 and KCS6 (Figure 3) was coherent with the reduction of alkane synthesis in NNMF exposed plants (see Figure 1A). The expression of LTPG1 has been shown to alter the ultrastructure of the cuticle layer of the stem epidermis and to cause the reduction of C29 alkane, which is a major component of cuticular waxes in the stems and siliques [30]. While KCS12 is known to affect wax biosynthesis [31], KCS1 has been shown to be involved in wax biosynthesis, but with limited effect on C29 alkanes [32]. In the bolting stage, the increased content of C18:1 and C18:2 in NNMF plants (see Figure 2B) was correlated to the upregulation of LTPG1 (Figure 3), whereas the increase of alkanes from C21 to C28 of NNMF plants (Figure 1B) was associated to the upregulation of KCS6, KCS8, KCS12, and LIP1 (Figure 3). In this stage of plant development, the reduction of C29 and C31 in NNMF plants was correlated to the downregulation of KCS5 (Figure 3). In the flowering stage, the consistent increase of alkanes from $\mathrm{C} 21$ to $\mathrm{C} 28$ in NNMF plants (see Figure 1C) was associated to the upregulation of ADS4, KCS5, KCS6, KCS8, KCS12, and LIP1 (Figure 3). Finally, in the seed-set stage, the almost undetectable alkane from $\mathrm{C} 1$ to $\mathrm{C} 28$ observed in both GMF and NNMF (Figure 1D) might depend on to the low expression and downregulation (in NNMF condition) of KCS1, KCS6, KCS8, KCS12, and LIP1 (Figure 3), whereas the increased content of C29 and C31 in NNMF plants (Figure 1D) correlated to the upregulation of KCS5 (Figure 3). However, we cannot exclude that owing to the are relatively stable nature of alkanes, these changes could be due to degradation by other enzymes. KCS6 has been correlated to changes in alkane composition during development [29] whereas the regulation of KCS5 (also known as CER60) has been shown to be involved in wax biosynthesis of long-chain alkanes [33]. Our results indicate a close association between the regulation of KCS5 and the production of C29 and C31 alkanes. LIP1 drives the synthesis of an active triacylglycerol (TAG) lipase and is capable of hydrolyzing long chain triacylglycerol [34]. The expression of LIP1 correlated with the synthesis of NNMF alkanes, particularly during bolting and flowering, where these wax constituents showed the highest content.

In a previous work we showed that photoreceptors, including cryptochrome, are involved in plant magnetoreception [15]. The functional modification of cryptochrome under NNMF conditions involves suppression of the phytohormone gibberellin (GA) biosynthesis [35]; and GA is involved in the modulation of fatty acid synthesis [36]. Therefore, we cannot exclude that plants may recruit signaling cascades that have evolved to respond to other stimuli and stress factors to give rise to magnetosensitive response.

Overall, such results indicated that the GMF modulates the lipid composition and metabolism in Arabidopsis and that reduction of the GMF to NNMF impairs the plant metabolism at different developmental stages of plants. These data are in line with previous developmental studies $[2,3,5]$. There was not a specific trend of gene expression during plant development; for instance, expression of KCS5 at NNMF-treated rosette and bolting stages was down-regulated, but upregulated at the flowering and seed-set stages. This might depend on the possibility that specific regulation of protein content as well as of the related enzymes activity occurs at different timing. The effect of higher induction on KCS5 at the flowering stage might result in C29 and C31 accumulation later at the seed-set stage. Nevertheless, we observed a link between induction of gene expression and metabolites accumulation. 


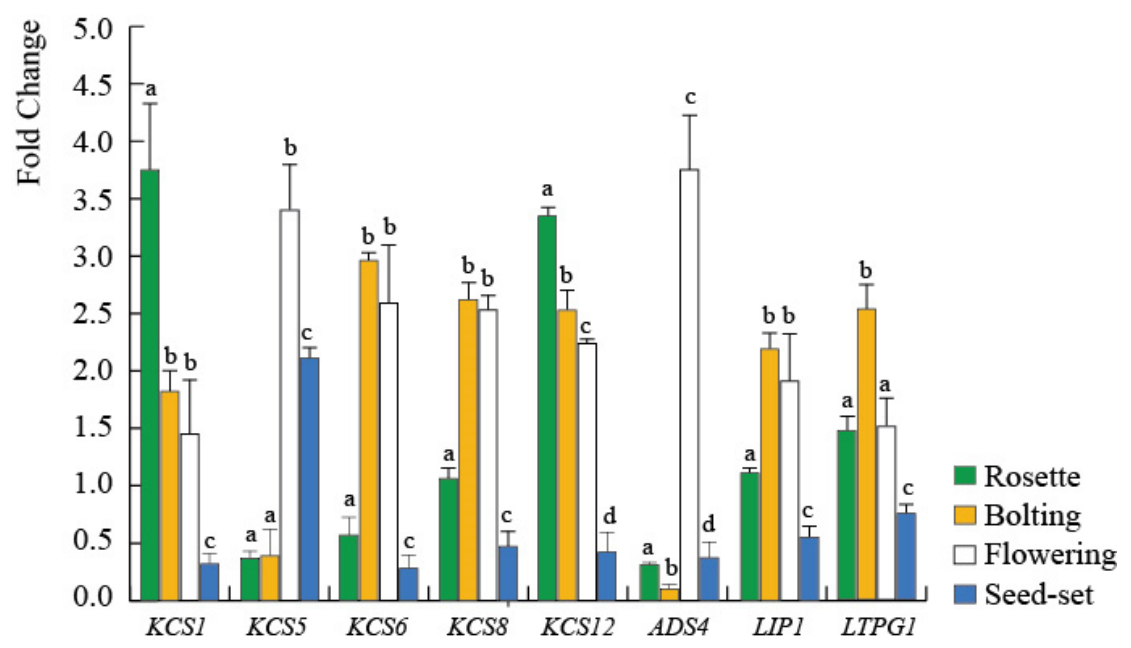

Figure 3. Time-course expression of genes in Arabidopsis thaliana exposed to NNMF conditions. Values are expressed as fold change with respect to control plants growing in GMF conditions. Bars represent standard deviation. Age of plants was the same as reported in Figures 1 and 2. For the same gene, different letters indicate significant $(p<0.05)$ differences between the different plant developmental stages.

\subsection{The GMF Modulates Plant Morphology}

Considering that several physiological processes linked to plant growth are under control of a complex network of lipid-mediated signaling [22], we investigate the role of the GMF on the growth of Arabidopsis plants at the different developmental stages by determining morphometry and parameters of shoots under NNMF conditions (Supplementary Table S1). Under NNMF condition, the leaf area index (LAI) was significantly $(p<0.05)$ reduced in the flowering and seed set developmental stages. As expected, the shoot length was reduced in NNMF treatment from bolting to seed-set, as previously observed [5], and was accompanied by a progressive reduction of both fresh weight and dry weight (Supplementary Table S1). We also confirmed that the flowering time was delayed under NNMF conditions (see page 3 of Supplementary Materials). The reduction of plant height under NNMF has been correlated to a delayed in flowering $[2,4,5]$ and our results imply also an involvement of the lipid metabolism in this process.

\subsection{The GMF Modulates Plant Homeostasis}

We recently demonstrated that NNMF condition impairs nutrient homeostasis in plants [7] at the early stage of plant development. In order to evaluate the effect of the GMF during plant development, we performed an elements composition analysis of plants at the rosette, bolting, flowering and seed-set stages.

At the rosette developmental stage, no significant variations were observed for the macronutrients, whereas iron $(\mathrm{Fe})$ was the only micronutrient showing a significant $(p<0.05)$ increased when compared with GMF conditions (Table 1). This finding is in agreement with our previous observations [7] and highlights the importance of the GMF for Fe homeostasis in plants during early development.

During bolting, a significant $(p<0.05)$ increase was observed only for the micronutrient cobalt (Co). Cobalt was found to increase its availability upon treatment of barley (Hordeum vulgare) with magnetic nanoparticles, with a significant increase with respect to control [37]. 
Table 1. Macro and micronutrients contents in different developmental stages from rosette to seed-set of Arabidopsis exposed to GMF (control) and NNMF. Data are expressed as mean value ( \pm standard deviation) from three independent biological replications. ${ }^{* *}$ Boldfaced figures indicate significant $(p<0.05)$ difference between GMF and NNMF.

\begin{tabular}{|c|c|c|c|c|c|c|c|c|}
\hline & \multicolumn{2}{|c|}{ Rosette } & \multicolumn{2}{|c|}{ Bolting } & \multicolumn{2}{|c|}{ Flowering } & \multicolumn{2}{|c|}{ Seed-Set } \\
\hline & GMF & NNMF & GMF & NNMF & GMF & NNMF & GMF & NNMF \\
\hline \multicolumn{9}{|c|}{ Macronutrients (mg/gDW) } \\
\hline $\mathrm{Na}$ & $3.47 \pm 1.67$ & $2.11 \pm 0.56$ & $1.15 \pm 0.09$ & $1.38 \pm 0.21$ & $1.00 \pm 0.87$ & $0.64 \pm 0.49$ & $0.43 \pm 0.09$ & $1.09 \pm 0.38$ \\
\hline $\mathrm{Mg}$ & $4.29 \pm 0.34$ & $4.04 \pm 0.04$ & $3.40 \pm 0.04$ & $3.74 \pm 0.63$ & $3.30 \pm 1.43$ & $2.36 \pm 1.78$ & $2.71 \pm 0.38$ & $3.84 \pm 0.48^{* *}$ \\
\hline $\mathrm{K}$ & $36.19 \pm 2.46$ & $36.76 \pm 2.50$ & $30.25 \pm 3.74$ & $31.75 \pm 5.42$ & $32.73 \pm 14.79$ & $21.93 \pm 16.43$ & $29.61 \pm 1.76$ & $34.46 \pm 2.33 * *$ \\
\hline $\mathrm{Ca}$ & $19.77 \pm 1.58$ & $20.11 \pm 1.09$ & $15.46 \pm 0.99$ & $17.28 \pm 2.58$ & $13.57 \pm 6.35$ & $9.93 \pm 7.68$ & $8.18 \pm 2.14$ & $16.26 \pm 3.86 * *$ \\
\hline $\mathrm{P}$ & $7.28 \pm 0.27$ & $7.64 \pm 0.28$ & $5.63 \pm 0.44$ & $5.62 \pm 0.77$ & $5.77 \pm 2.07$ & $4.08 \pm 3.03$ & $6.32 \pm 0.43$ & $5.23 \pm 1.40$ \\
\hline \multicolumn{9}{|c|}{ Micronutrients $(\mu \mathrm{g} / \mathrm{gDW})$} \\
\hline $\mathrm{Mn}$ & $23.59 \pm 1.06$ & $27.29 \pm 2.84$ & $19.75 \pm 0.70$ & $20.14 \pm 10.07$ & $17.84 \pm 4.96$ & $10.98 \pm 7.97$ & $18.23 \pm 1.33$ & $21.99 \pm 1.37^{* *}$ \\
\hline $\mathrm{Fe}$ & $334.24 \pm 57.52$ & $610.57 \pm 153.40 * *$ & $287.77 \pm 82.51$ & $281.50 \pm 166.94$ & $126.5 \pm 69.09$ & $112.94 \pm 23.12$ & $90.93 \pm 23.33$ & $142.47 \pm 35.31$ \\
\hline Co & $0.18 \pm 0.02$ & $0.11 \pm 0.09$ & $0.13 \pm 0.04$ & $0.20 \pm 0.02 * *$ & $0.07 \pm 0.04$ & $0.12 \pm 0.02$ & $0.14 \pm 0.02$ & $0.07 \pm 0.04 * *$ \\
\hline $\mathrm{Ni}$ & $3.67 \pm 0.97$ & $5.55 \pm 3.53$ & $2.31 \pm 0.70$ & $3.89 \pm 2.34$ & $2.37 \pm 2.10$ & $1.69 \pm 1.15$ & $1.66 \pm 0.54$ & $3.25 \pm 0.15^{* *}$ \\
\hline $\mathrm{Cu}$ & $15.02 \pm 2.37$ & $17.90 \pm 5.63$ & $14.07 \pm 1.88$ & $10.18 \pm 2.29$ & $12.70 \pm 10.50$ & $8.38 \pm 6.14$ & $9.98 \pm 1.69$ & $11.87 \pm 1.12$ \\
\hline $\mathrm{Zn}$ & $318.15 \pm 87.91$ & $346.33 \pm 267.53$ & $228.44 \pm 85.19$ & $194.07 \pm 42.44$ & $456.97 \pm 672.99$ & $77.27 \pm 62.36$ & $76.88 \pm 11.29$ & $82.09 \pm 4.83$ \\
\hline Mo & $12.63 \pm 10.32$ & $7.38 \pm 2.33$ & $3.69 \pm 1.08$ & $4.92 \pm 1.84$ & $2.93 \pm 1.37$ & $2.21 \pm 1.70$ & $1.77 \pm 0.30$ & $3.31 \pm 0.79 * *$ \\
\hline
\end{tabular}


While plants did not display variation in the nutritional status during the flowering stage, NNMF exposure strongly affected the content of several nutrients in leaves of plants at the seed set developmental stage. In particular, macronutrients like magnesium $(\mathrm{Mg})$, potassium $(\mathrm{K})$ and calcium (Ca) showed a significant $(p<0.05)$ increase in their content with respect to GMF-exposed plants. Moreover, micronutrients such as manganese (Mn), molybdenum (Mo) and nickel (Ni) showed a significantly $(p<0.05)$ higher accumulation under NNMF when compared to plants grown in GMF conditions. Interestingly, during seed set the content of Co was significantly reduced by exposure to NNMF (Table 1).

The inorganic elements content profile of leaves is considered to be a signature of the nutrient status of plants under stress conditions [38,39]. Along with morphometric data, ionomic analysis highlight that MF variations are perceived as a stress condition by plants in a developmental stage-dependent manner.

\subsection{The GMF Modulation Highlights the Link between Lipid Metabolism and Plant Nutrition}

A link between the mineral nutrient status and the lipid metabolism in higher plants has been demonstrated. Indeed, several studies reported upregulation of 3-Ketoacyl-CoA synthase genes including KCS12, KC17 and KCS21, in Chlamydomonas and rice plant under N, Fe, Pi and S starvation [40,41], as well as an increase of several fatty acids such as 16:0, 16:1, 18:0, 18:1, 18:2 and 18:3 in pea leaves under Fe deficiency condition [42]. Fe excess treatment leads to alteration of lipid metabolism, with upregulation of protease inhibitor/seed storage/lipid transfer (LTP) and 3-ketoacyl-CoA synthase (KSC) proteins [43]. Therefore, under NNMF conditions, the observed increase of Fe content might be associated to the upregulation of KCS12 and LTPG1 found in leaves of Arabidopsis at the rosette stage. In addition, the variation of some macronutrients and micronutrients at the seed-set stage might be linked to the upregulation of KCS5 and downregulation of ADS4, LIP1 and LTPG1 genes. In fact, a down regulation of three KCS genes, such as 3-ketoacyl-CoA synthase-12, -17 and -21 , has been observed in Mg-deficient Citrus sinensis plants [44]. Furthermore, Mn-deficient Arabidopsis plants displayed upregulation of gene encoding for the fatty acid desaturase family protein ADS4 [45]. Several lipase genes were upregulated under P starvation and down regulated under K-deficient condition in rice [46] and Citrus sinensis leaves [44].

Additionally, Wu et. al. [47] reported that, molybdenum application can induce alteration of fatty acids of thylakoid membranes particularly increasing of C16:0, C18:2 and induce low temperature in wheat.

\section{Materials and Methods}

\subsection{Plant Materials and Growth Conditions}

Arabidopsis thaliana ecotype Columbia 0 (Col-0) were vernalized at $4{ }^{\circ} \mathrm{C}$ for $72 \mathrm{~h}$ in dark condition in order to synchronize the plants. Seeds were then sown in $8 \mathrm{~cm}$ diameter polyethylene pots with soil prepared with a mixture of peat and vermiculite ( 2 parts of peat and 1 part of vermiculite). For each developmental stage, at least 10 pots for both control and NNMF exposed plants were assayed and the experiment was repeated at least three times (see also Supplementary Figure S1). Sown pots (in both GMF and NNMF exposed plants) were exposed to homogenous irradiation from a high pressure sodium lamp source (Grolux 600W, SYLVANIA, Antwerpen, Belgium) at $200 \mu \mathrm{mol} \mathrm{m}{ }^{-2} \mathrm{~s}^{-1}$, $22{ }^{\circ} \mathrm{C}\left( \pm 1.5^{\circ} \mathrm{C}\right)$ with a photoperiod of $14 \mathrm{~h}$ light and $10 \mathrm{~h}$ darkness. Control experiments (GMF) were performed in the same lab at a distance of $6 \mathrm{~m}$ from the NNMF controlling system and the measured levels of power-line frequency $(50 \mathrm{~Hz})$, light and temperature $\left(22^{\circ} \mathrm{C}\right)$ of both controls and treatments were similar. Treated plants were grown inside the NNMF (see below) controlling system. All experiments were performed under normal gravity. Because NNMF delays plant flowering [5], sampling timing was set on control plants (i.e., plants exposed to GMF) and was performed at 15 days after sowing (DAS) during the early vegetative stage of development, at 21 DAS during the bolting stage of development, at 30 DAS at full bloom and after 35 DAS during the seed-set stage of plant 
development (see also Supplementary Figure S1). For Morphological analysis, each time course pictures were collected to measure leaf area index and stem length. Leaf area index (LAI) was measured by dividing the leaf area by the pot area. Stem length was measured from the base to the tip of the flowering stem. Fresh and dry biomass were also calculated.

\subsection{GMF Control System}

The GMF B values of control plants were in line with the values of the Northern hemisphere [48]. The geographical coordinates of the laboratory were $45^{\circ} 0^{\prime} 59^{\prime \prime} \mathrm{N}$ and $7^{\circ} 36^{\prime} 58^{\prime \prime} \mathrm{E}$ and the $\mathrm{B}$ value measured was $40.3 \mu \mathrm{T}$ with an inclination of +57 degrees. Near-null magnetic field (NNMF) was obtained by three couples of orthogonal Helmholtz coils connected to three direct current (DC) power supplies as previously detailed [5]. Real-time monitoring of the magnetic field inside the NNMF controlling system was achieved as previously described [5]. Polyethylene pots containing Arabidopsis seeds were exposed either to NNMF or to GMF. Double-blinded experiments were performed and a quantitative descriptions of applied fields and their spatial distributions along with measurement uncertainties were as fully described earlier [3,5].

\subsection{Fatty Acid and Cuticular Alkane Chemical Composition}

For both FA and alkane analyses and each time point, Arabidopsis plants exposed to either GMF or NNMF were harvested and immediately frozen in liquid nitrogen and the plant material was stored at $-80{ }^{\circ} \mathrm{C}$. The plant material from pooled pots was then used for extractions.

\subsubsection{Fatty Acid Analysis}

The pooled collected leaves from each developmental stage (from 20 to $40 \mathrm{~g}$, depending on the developmental stage) were extracted using cyclohexane (1:10, weight to volume ratio) and then esterified with boron tri-fluoride $(10 \% w / v$ in methanol). Fifty $\mu \mathrm{g}$ heptadecanoic acid (C17:0) were added as internal standard [49]. The fatty acid methyl esters (FAME) were obtained by acid catalysis according to Christie and Han [50] and were dehydrated with anhydrous $\mathrm{MgSO}_{4}$. FAME identification and quantification was performed by gas chromatography-mass spectrometry (GC-MS) (5975T, Agilent Technologies, Santa Clara, CA, USA) and by gas chromatography flame ionization detector (GC-FID) (GC-2010 Plus, SHIMADZU, Kyoto, Japan), respectively, as reported earlier [49]. At least three technical replicates were run for each developmental stage of plants exposed to either GMF or NNMF.

\subsubsection{Cuticular Alkane Analysis}

The pooled collected leaves from each developmental stage (from 20 to $40 \mathrm{~g}$, depending on the developmental stage) were extracted with a mixture of $5 \mathrm{ml}$ pentane:hexane (5:1) for $60 \mathrm{~s} ; 30 \mu \mathrm{g}$ of n-tritriacontane were added as internal standard. The extract was concentrated by a gentle stream of gaseous $\mathrm{N}_{2}$, passed through a column of anhydrous $\mathrm{MgSO}_{4}$ and then analysed by GC-FID and GC-MS as previously described [51]. An average of three injections was done for each sample. The following abbreviations and the diagnostic ions $(\mathrm{m} / \mathrm{z})$ of the identified alkanes were: C21 n-heneicosane (296), C22 n-docosane (310), C23 n-tricosane (324), C24 n-tetracosane (338), C25 n-pentacosane (352), C26-hexacosane (366), C27 n-heptacosane (380), C28 n-octacosane (394), C29 n-nonacosane (408), C30 n-triacontane (422), C31 n- hentriacontane (436).

\subsection{Ionome Analysis}

For ionomic analyses, leaves were collected from each time point of Arabidopsis plants exposed to either GMF or NNMF, and dried in an aerate oven at $70{ }^{\circ} \mathrm{C}$ for four days. Samples dry weight were measured and digested by using $65 \% \mathrm{HNO}_{3}$ at $120{ }^{\circ} \mathrm{C}$. The mineralized samples were transferred into polypropylene test tubes and diluted 1:40 in MILLI-Q water. Finally, the concentration 
of metal elements was measured by inductively coupled plasma-mass Spectrometry ICP-MS (BRUKER Aurora-M90 ICP-MS) as previously described [7].

\subsection{RNA Isolation and cDNA Synthesis}

For each developmental stage, $100 \mathrm{mg}$ of frozen samples exposed to either GMF or NNMF were ground separately in liquid nitrogen with mortar and pestle. Total RNA was isolated using the Machery-Nagel RNA Isolation mini Kit (Machery-Nagel, Duren, Germany), and RNase-free DNAse, according to the manufacturer's protocols. RNA quality and quantity were checked as previously described [3]. cDNA was synthesized starting from $1 \mu \mathrm{g}$ RNA using the High Capacity cDNA Reverse Transcription kit (Applied Biosystem, Foster City, CA, USA), according to the manufacturer's recommendations. Reaction mixtures were incubated at $25^{\circ} \mathrm{C}$ for $10 \mathrm{~min}, 37^{\circ} \mathrm{C}$ for $2 \mathrm{~h}$, and $85^{\circ} \mathrm{C}$ for $5 \mathrm{~min}$.

\subsection{Quantitative Real-Time PCR ( $q P C R$ )}

All qPCR experiments were performed on a Stratagene Mx3000P Real-Time System (La Jolla, CA, USA) using SYBR green I with $\operatorname{ROX}^{\circledR}$ as an internal loading standard. The reaction was performed with $25 \mu \mathrm{L}$ of mixture consisting of $12.5 \mu \mathrm{L}$ of $2 X_{\text {Maxima }}{ }^{\mathrm{TM}}$ SYBR Green/ROX qPCR Master Mix (Fermentas International, Burlington, ON, Canada), $0.5 \mu \mathrm{L}$ 1:5 diluted cDNA and $100 \mathrm{nM}$ primers (Integrated DNA Technologies, Coralville, IA, USA). Controls included non-RT controls (using total RNA without reverse transcription to monitor for genomic DNA contamination) and non-template controls (water template). All primers were designed using Primer 3 software [52]. Primers used for real-time PCR are reported in Supplementary Table S2. Specifically, PCR cycles were $10 \mathrm{~min}$ at $95^{\circ} \mathrm{C}, 45$ cycles of $15 \mathrm{~s}$ at $95^{\circ} \mathrm{C}, 20 \mathrm{~s}$ at $57^{\circ} \mathrm{C}$, and $30 \mathrm{~s}$ at $72^{\circ} \mathrm{C}$ and all runs were followed by a melting curve analysis with the following gradient: $1 \mathrm{~min}$ at $95^{\circ} \mathrm{C}, 30 \mathrm{~s}$ at $55^{\circ} \mathrm{C}, 30 \mathrm{~s}$ at $95^{\circ} \mathrm{C}$ for $A t 1 g 06350$, $\triangle 9$ DESATURASE 4 (ADS4); At1g27950, GLYCOSYLPHOSPHATIDYLINOSITOL-ANCHORED LIPID PROTEIN TRANSFER 1 (LTPG1); At1g01120, 3-KETOACYL-COA SYNTHASE 1, (KCS1); At1g25450, 3-KETOACYL-COA SYNTHASE 5 (KCS5); At1g68530, 3-KETOACYL-COA SYNTHASE 6 (KCS6); At2g15090, 3-KETOACYL-COA SYNTHASE 8 (KCS8); At2g28630, 3-KETOACYL-COA SYNTHASE 12 (KCS12); At2g15230, LIPASE 1 (LIP1). Four different reference genes At2g37620, ACTIN1 (ACT1); At5g19510, ELONGATION FACTOR 1B ALPHA-SUBUNIT 2 (eEF1Balpha2); At1g13440, CYTOPLASMIC GLYCERALDEHYDE-3-PHOSPHATE DEHYDROGENASE (GAPC2); and At1g51710, UBIQUITIN SPECIFIC PROTEASE 6 (UBP6), which were used to normalize the results of the qPCR. The best performing of these four genes was selected using Normfinder software (MOMA, Aarhus, Denmark) [53]. Normfinder analysis revealed that the most stable gene was eEF1Balpha2.

All amplification plots were analyzed with the Mx3000P software to obtain Ct values. qPCR data are expressed as fold change of NNMF with respect to control plants growing in GMF conditions.

\subsection{Statistical Analyses}

The data obtained from lipidomics and qPCR were treated by using Systat 10 (Systat Software, San Jose, CA, USA). Mean values were calculated along with the standard deviation (SD). Paired $t$ test and Bonferroni adjusted probability were used to assess the difference between treatments and the control. ANOVA was calculated in the comparative analysis of plant development data and a Tukey post hoc test was assessed.

\section{Conclusions}

Our results show that the GMF modulates Arabidopsis thaliana nutrition and lipid composition and this effect is related to the developmental stage of plants and that its presence is necessary for a normal development and metabolism. By reducing the GMF to NNMF we observed an increased amount of epicuticular alkanes and a modulation of FAs unsaturation. These events were associated to alteration in the nutritional status of NNMF exposed plants. Homeostasis and lipid composition are sensitive to several abiotic stresses [54-56] and our results suggest that the alteration of the GMF might be 
considered a source of abiotic stress. For instance, plants respond to varying MF by increasing reactive oxygen species (ROS) production $[1,9,16,57,58]$, and ROS generation is a typical response to stress conditions in plants [59]. Our working hypothesis is that the GMF, just like light, gravity and touch can be perceived by plants and variations in the GMF might cause a stress condition (see also [60]). By considering that the GMF varies with latitude, we may expect variations in plants exposed to different GMF values. The evidence of differential root and shoot [57] and both light-dependent and light-independent responses to GMF variations $[15,17]$ point to an hypothetical mechanism that might rely on different effectors. The search for intracellular effectors of GMF will surely provide more insights on plant magnetoreception.

Supplementary Materials: The following are available online at http://www.mdpi.com/2223-7747/9/12/1729/s1, Table S1: Direct comparison of morphological and developmental parameters between Arabidopsis thaliana exposed to either GMF or NNMF, Table S2: primers used in qPCR analyses. Figure S1, time couse exposure of Arabidopsis to NNMF and GMF conditions.

Author Contributions: Conceptualization, M.E.M.; methodology, M.E.M., M.I. and G.V.; validation, M.E.M., M.I. and G.V.; formal analysis, M.I. and G.V.; investigation, M.I. and G.V.; resources, M.E.M. and G.V.; data curation, M.E.M., M.I. and G.V.; writing—original draft preparation, M.E.M., M.I. and G.V.; writing—review and editing, M.E.M., M.I. and G.V.; visualization, M.E.M., M.I. and G.V.; supervision, M.E.M.; project administration, M.E.M.; funding acquisition, M.E.M. and G.V. All authors have read and agreed to the published version of the manuscript.

Funding: This research was funded by the University of Turin, local research grants to M.E.M. and to M.I. by the Doctorate School of Pharmaceutical and Biomolecular Sciences of the University of Turin.

Conflicts of Interest: The authors declare no conflict of interest.

\section{References}

1. Maffei, M.E. Magnetic field effects on plant growth, development, and evolution. Front. Plant Sci. 2014, 5, 445. [CrossRef] [PubMed]

2. Xu, C.X.; Wei, S.F.; Lu, Y.; Zhang, Y.X.; Chen, C.F.; Song, T. Removal of the local geomagnetic field affects reproductive growth in arabidopsis. Bioelectromagnetics 2013, 34, 437-442. [CrossRef] [PubMed]

3. Agliassa, C.; Maffei, M.E. Reduction of geomagnetic field (gmf) to near null magnetic field (nnmf) affects some arabidopsis thaliana clock genes amplitude in a light independent manner. J. Plant Physiol. 2019, 232, 23-26. [CrossRef] [PubMed]

4. Xu, C.X.; Yin, X.; Lv, Y.; Wu, C.Z.; Zhang, Y.X.; Song, T. A near-null magnetic field affects cryptochrome-related hypocotyl growth and flowering in arabidopsis. Adv. Space Res. 2012, 49, 834-840. [CrossRef]

5. Agliassa, C.; Narayana, R.; Bertea, C.M.; Rodgers, C.T.; Maffei, M.E. Reduction of the geomagnetic field delays arabidopsis thaliana flowering time through downregulation of flowering-related genes. Bioelectromagnetics 2018, 39, 361-374. [CrossRef]

6. Narayana, R.; Fliegmann, J.; Paponov, I.; Maffei, M.E. Reduction of geomagnetic field (gmf) to near null magnetic field (nnmf) affects Arabidopsis thaliana root mineral nutrition. Life Sci. Space Res. 2018, 19, 43-50. [CrossRef]

7. Islam, M.; Maffei, M.E.; Vigani, G. The geomagnetic field is a contributing factor for an efficient iron uptake in Arabidopsis thaliana. Front. Plant Sci. 2020, 11, 325. [CrossRef]

8. Guo, J.P.; Wan, H.Y.; Matysik, J.; Wang, X.J. Recent advances in magnetosensing cryptochrome model systems. Acta Chim. Sin. 2018, 76, 597-604. [CrossRef]

9. Pooam, M.; Arthaut, L.D.; Burdick, D.; Link, J.; Martino, C.F.; Ahmad, M. Magnetic sensitivity mediated by the arabidopsis blue-light receptor cryptochrome occurs during flavin reoxidation in the dark. Planta 2019, 249, 319-332. [CrossRef]

10. Kornig, A.; Winklhofer, M.; Baumgartner, J.; Gonzalez, T.P.; Fratzl, P.; Faivre, D. Magnetite crystal orientation in magnetosome chains. Adv. Funct. Mater. 2014, 24, 3926-3932. [CrossRef]

11. Kempster, R.M.; McCarthy, I.D.; Collin, S.P. Phylogenetic and ecological factors influencing the number and distribution of electroreceptors in elasmobranchs. J. Fish Biol. 2012, 80, 2055-2088. [CrossRef] [PubMed]

12. Vanderstraeten, J.; Gailly, P.; Malkemper, E.P. Low-light dependence of the magnetic field effect on cryptochromes: Possible relevance to plant ecology. Front. Plant Sci. 2018, 9, 121. [CrossRef] [PubMed] 
13. Xu, C.; Li, Y.; Yu, Y.; Zhang, Y.; Wei, S. Suppression of arabidopsis flowering by near-null magnetic field is affected by light. Bioelectromagnetics 2015, 36, 476-479. [CrossRef] [PubMed]

14. Dhiman, S.K.; Galland, P. Effects of weak static magnetic fields on the gene expression of seedlings of arabidopsis thaliana. J. Plant Physiol. 2018, 231, 9-18. [CrossRef]

15. Agliassa, C.; Narayana, R.; Christie, J.M.; Maffei, M.E. Geomagnetic field impacts on cryptochrome and phytochrome signaling. J. Photochem. Photobiol. B 2018, 185, 32-40. [CrossRef]

16. Hammad, M.; Albaqami, M.; Pooam, M.; Kernevez, E.; Witczak, J.; Ritz, T.; Martino, C.; Ahmad, M. Cryptochrome mediated magnetic sensitivity in arabidopsis occurs independently of light-induced electron transfer to the flavin. Photochem. Photobiol. Sci. 2020, 19, 341-352. [CrossRef]

17. Xu, C.; Lv, Y.; Chen, C.; Zhang, Y.; Wei, S. Blue light-dependent phosphorylations of cryptochromes are affected by magnetic fields in arabidopsis. Adv. Space Res. 2014, 53, 1118-1124. [CrossRef]

18. Ahmad, M.; Cashmore, A.R. The blue-light receptor cryptochrome 1 shows functional dependence on phytochrome a or phytochrome b in arabidopsis thaliana. Plant J. 1997, 11, 421-427. [CrossRef]

19. Troncoso-Ponce, M.A.; Nikovics, K.; Marchive, C.; Lepiniec, L.; Baud, S. New insights on the organization and regulation of the fatty acid biosynthetic network in the model higher plant Arabidopsis thaliana. Biochimie 2016, 120, 3-8. [CrossRef]

20. Hegebarth, D.; Jetter, R. Cuticular waxes of arabidopsis thaliana shoots: Cell-type-specific composition and biosynthesis. Plants 2017, 6, 19. [CrossRef]

21. Upchurch, R.G. Fatty acid unsaturation, mobilization, and regulation in the response of plants to stress. Biotechnol. Lett. 2008, 30, 967-977. [CrossRef] [PubMed]

22. Hou, Q.C.; Ufer, G.D.; Bartels, D. Lipid signalling in plant responses to abiotic stress. Plant Cell Environ. 2016, 39, 1029-1048. [CrossRef] [PubMed]

23. Novitskaya, G.V.; Kocheshkova, T.; Novitskii, Y.I. The effects of a weak permanent magnetic field on the lipid composition and content in the onion leaves of various ages. Russ. J. Plant Physiol. 2006, 53, 638-648. [CrossRef]

24. Novitskaya, G.V.; Molokanov, D.; Kocheshkova, T.; Novitskii, Y.I. Effect of weak constant magnetic field on the composition and content of lipids in radish seedlings at various temperatures. Russ. J. Plant Physiol. 2010, 57, 52-61. [CrossRef]

25. Novitskii, Y.I.; Novitskaya, G.V.; Serdyukov, Y.A.; Kocheshkova, T.K.; Molokanov, D.R.; Dobrovolskii, M.V. Influence of weak permanent magnetic field on lipid peroxidation in radish seedlings. Russ. J. Plant Physiol. 2015, 62, 375-380. [CrossRef]

26. Novitskii, Y.I.; Novitskaya, G.V.; Serdyukov, Y.A.; Kocheshkova, T.K.; Dobrovolskii, M.V. Lipid composition and content in the seeds of radish plants of different magnetic orientation grown in weak permanent magnetic field. Russ. J. Plant Physiol. 2014, 61, 409-418. [CrossRef]

27. Novitskii, Y.I.; Novitskaya, G.V.; Molokanov, D.R.; Serdyukov, Y.A.; Yusupova, I.U. The influence of a weak horizontal permanent magnetic field on the composition and content of lipids in lettuce leaves. Biol. Bull. 2015, 42, 411-418. [CrossRef]

28. Dennison, T.; Qin, W.M.; Loneman, D.M.; Condon, S.G.F.; Lauter, N.; Nikolau, B.J.; Yandeau-Nelson, M.D. Genetic and environmental variation impact the cuticular hydrocarbon metabolome on the stigmatic surfaces of maize. BMC Plant Biol. 2019, 19, 16. [CrossRef]

29. Busta, L.; Hegebarth, D.; Kroc, E.; Jetter, R. Changes in cuticular wax coverage and composition on developing arabidopsis leaves are influenced by wax biosynthesis gene expression levels and trichome density. Planta 2017, 245, 297-311. [CrossRef]

30. Kim,H.; Lee, S.B.; Kim,H.J;;Min, M.K.; Hwang, I.;Suh, M.C.Characterization of glycosylphosphatidylinositol-anchored lipid transfer protein 2 (ltpg2) and overlapping function between ltpg/ltpg1 and ltpg2 in cuticular wax export or accumulation in arabidopsis thaliana. Plant Cell Physiol. 2012, 53, 1391-1403. [CrossRef]

31. Suh, M.C.; Samuels, A.L.; Jetter, R.; Kunst, L.; Pollard, M.; Ohlrogge, J.; Beisson, F. Cuticular lipid composition, surface structure, and gene expression in arabidopsis stem epidermis. Plant Physiol. 2005, 139, 1649-1665. [CrossRef] [PubMed]

32. Todd, J.; Post-Beittenmiller, D.; Jaworski, J.G. Kcs1 encodes a fatty acid elongase 3-ketoacyl-coa synthase affecting wax biosynthesis in Arabidopsis thaliana. Plant J. 1999, 17, 119-130. [CrossRef] [PubMed] 
33. Tresch, S.; Heilmann, M.; Christiansen, N.; Looser, R.; Grossmann, K. Inhibition of saturated very-long-chain fatty acid biosynthesis by mefluidide and perfluidone, selective inhibitors of 3-ketoacyl-coa synthases. Phytochemistry 2012, 76, 162-171. [CrossRef] [PubMed]

34. Karim, E.K.; Stephanie, B.; Emilia, O.; Anne-Marie, G.; Natalie, F.; Vincent, A. Identification and characterization of a triacylglycerol lipase in arabidopsis homologous to mammalian acid lipases. FEBS Lett. 2005, 579, 6067-6073.

35. Xu, C.; Yu, Y.; Zhang, Y.; Li, Y.; Wei, S. Gibberellins are involved in effect of near-null magnetic field on arabidopsis flowering. Bioelectromagnetics 2017, 38, 1-10. [CrossRef]

36. Jusoh, M.; Loh, S.H.; Aziz, A.; Cha, T.S. Gibberellin promotes cell growth and induces changes in fatty acid biosynthesis and upregulates fatty acid biosynthetic genes in chlorella vulgaris umt-m1. Appl. Biochem. Biotechnol. 2019, 188, 450-459. [CrossRef]

37. Tombuloglu, H.; Slimani, Y.; Alshammari, T.; Tombuloglu, G.; Almessiere, M.; Baykal, A.; Ercan, I.; Ozcelik, S.; Demirci, T. Magnetic behavior and nutrient content analyses of barley (Hordeum vulgare L.) tissues upon cond0.2fe1.8o4 magnetic nanoparticle treatment. J. Soil Sci. Plant Nutr. 2020, 20, 357-366. [CrossRef]

38. Pii, Y.; Cesco, S.; Mimmo, T. Shoot ionome to predict the synergism and antagonism between nutrients as affected by substrate and physiological status. Plant Physiol. Biochem. 2015, 94, 48-56. [CrossRef]

39. Martin-Sanchez, L.; Ariotti, C.; Garbeva, P.; Vigani, G. Investigating the effect of belowground microbial volatiles on plant nutrient status: Perspective and limitations. J. Plant Interact. 2020, 15, 188-195. [CrossRef]

40. Hernandez-Torres, A.; Zapata-Morales, A.L.; Alfaro, A.E.O.; Soria-Guerra, R.E. Identification of gene transcripts involved in lipid biosynthesis in chlamydomonas reinhardtii under nitrogen, iron and sulfur deprivation. World J. Microbiol. Biotechnol. 2016, 32, 55. [CrossRef]

41. Wasaki, J.; Yonetani, R.; Kuroda, S.; Shinano, T.; Yazaki, J.; Fujii, F.; Shimbo, K.; Yamamoto, K.; Sakata, K.; Sasaki, T.; et al. Transcriptomic analysis of metabolic changes by phosphorus stress in rice plant roots. Plant Cell Environ. 2003, 26, 1515-1523. [CrossRef]

42. Abadia, A.; Ambardbretteville, F.; Remy, R.; Tremolieres, A. Iron-deficiency in pea leaves—Effect on lipid composition and synthesis. Physiol. Plant. 1988, 72, 713-717. [CrossRef]

43. Sudre, D.; Gutierrez-Carbonell, E.; Lattanzio, G.; Rellan-Alvarez, R.; Gaymard, F.; Wohlgemuth, G.; Fiehn, O.; Alvarez-Fernandez, A.; Zamarreno, A.M.; Bacaicoa, E.; et al. Iron-dependent modifications of the flower transcriptome, proteome, metabolome, and hormonal content in an arabidopsis ferritin mutant. J. Exp. Bot. 2013, 64, 2665-2688. [CrossRef] [PubMed]

44. Yang, L.T.; Zhou, Y.F.; Wang, Y.Y.; Wu, Y.M.; Ye, X.; Guo, J.X.; Chen, L.S. Magnesium deficiency induced global transcriptome change in citrus sinensis leaves revealed by rna-seq. Int. J. Mol. Sci. 2019, 20, 3129. [CrossRef]

45. Yang, T.J.W.; Perry, P.J.; Ciani, S.; Pandian, S.; Schmidt, W. Manganese deficiency alters the patterning and development of root hairs in arabidopsis. J. Exp. Bot. 2008, 59, 3453-3464. [CrossRef]

46. Shankar, A.; Singh, A.; Kanwar, P.; Srivastava, A.K.; Pandey, A.; Suprasanna, P.; Kapoor, S.; Pandey, G.K. Gene expression analysis of rice seedling under potassium deprivation reveals major changes in metabolism and signaling components. PLoS ONE 2013, 8, e70321. [CrossRef]

47. Wu, S.W.; Wei, S.Q.; Hu, C.X.; Tan, Q.L.; Huang, T.W.; Sun, X.C. Molybdenum-induced alteration of fatty acids of thylakoid membranes contributed to low temperature tolerance in wheat. Acta Physiol. Plant. 2017, 39, 237. [CrossRef]

48. Kauristie, K.; Morschhauser, A.; Olsen, N.; Finlay, C.C.; McPherron, R.L.; Gjerloev, J.W.; Opgenoorth, H.J. On the usage of geomagnetic indices for data selection in internal field modelling. Space Sci. Rev. 2017, 206, 61-90. [CrossRef]

49. Mannino, G.; Gentile, C.; Maffei, M.E. Chemical partitioning and DNA fingerprinting of some pistachio (pistacia vera 1.) varieties of different geographical origin. Phytochemistry 2019, 160, 40-47. [CrossRef]

50. Christie, W.W.; Han, X. Lipid Analysis, 4th ed.; Woodhead Publishing: Oxford, UK, 2010.

51. Maffei, M. Chemotaxonomic significance of leaf wax alkanes in the gramineae. Biochem. Syst. Ecol. 1996, 24, 53-64. [CrossRef]

52. Rozen, S.; Skaletsky, H. Primer3 on the www for general users and for biologist programmers. In Bioinformatics Methods and Protocols; Misener, S., Krawetz, S.A., Eds.; Humana Press: Totowa, NJ, USA, 2000; pp. 365-386. 
53. Andersen, C.L.; Jensen, J.L.; Orntoft, T.F. Normalization of real-time quantitative reverse transcription-pcr data: A model-based variance estimation approach to identify genes suited for normalization, applied to bladder and colon cancer data sets. Cancer Res. 2004, 64, 5245-5250. [CrossRef] [PubMed]

54. Martin, L.B.B.; Romero, P.; Fich, E.A.; Domozych, D.S.; Rose, J.K.C. Cuticle biosynthesis in tomato leaves is developmentally regulated by abscisic acid. Plant Physiol. 2017, 174, 1384-1398. [CrossRef] [PubMed]

55. Scalabrin, E.; Radaelli, M.; Rizzato, G.; Bogani, P.; Buiatti, M.; Gambaro, A.; Capodaglio, G. Metabolomic analysis of wild and transgenic Nicotiana langsdorffii plants exposed to abiotic stresses: Unraveling metabolic responses. Anal. Bioanal. Chem. 2015, 407, 6357-6368. [CrossRef] [PubMed]

56. Chang, Y.N.; Zhu, C.; Jiang, J.; Zhang, H.M.; Zhu, J.K.; Duan, C.G. Epigenetic regulation in plant abiotic stress responses. J. Integr. Plant Biol. 2020, 62, 563-580. [CrossRef]

57. Bertea, C.M.; Narayana, R.; Agliassa, C.; Rodgers, C.T.; Maffei, M.E. Geomagnetic field (gmf) and plant evolution: Investigating the effects of gmf reversal on Arabidospis thaliana development and gene expression. J. Visual. Exp. 2015, 105, e53286. [CrossRef]

58. Ahmad, M. Photocycle and signaling mechanisms of plant cryptochromes. Curr. Opin. Plant Biol. 2016, 33, 108-115. [CrossRef]

59. Chen, Q.H.; Yang, G.W. Signal function studies of ros, especially rboh-dependent ros, in plant growth, development and environmental stress. J. Plant Growth Regul. 2020, 39, 157-171. [CrossRef]

60. Radhakrishnan, R. Magnetic field regulates plant functions, growth and enhances tolerance against environmental stresses. Physiol. Mol. Biol. Plants 2019, 25, 1107-1119. [CrossRef]

Publisher's Note: MDPI stays neutral with regard to jurisdictional claims in published maps and institutional affiliations. 